Tohoku J. Exp. Med., 2005, 207, 239-242

Case Report

\title{
Spinal Anesthesia in a Patient with Severe Thoracolumbar Kyphoscoliosis
}

\author{
Gurayten Ozyurt, Elif Basagan-Mogol, Hulya Bilgin and Oguz Tokat \\ Department of Anesthesiology and Reanimation, Uludag University Medical \\ School, Gorukle, Bursa, Turkey
}

Ozyurt, G., Basagan-Mogol, E., Bilgin, H. and Tokat, O. Spinal Anesthesia in a Patient with Severe Thoracolumbar Kyphoscoliosis. Tohoku J. Exp. Med., 2005, 207 (3), 239-242 — Patients with spine abnormalities, present unique challenges to the health care provider responsible for administrating sedation and anesthesia during surgical and technical procedures. Spinal deformities may cause difficulties with both tracheal intubation and regional anesthesia. This report describes the anesthetic management for two urological operations that were performed in a patient with extremely severe thoracolumbar kyphoscoliosis. After examining the risk factors, spinal block by injecting single dose local anesthetic solution to the intratechal space was chosen to provide anesthesia. It has been suggested that hyperbaric solution, which is of high density compared with cerebrospinal fluid, can safely produce blocks for many operations under spinal anesthesia. In the first procedure, intrathecal injection of $6 \mathrm{mg}$ hyperbaric bupivacaine, a local anesthetic solution (1.2 $\mathrm{ml}$ total volume), resulted in inadequate motor and sensory blockade, but the successful motor and sensory blockade at the level of Th10 was achieved in a second attempt with $6.25 \mathrm{mg}$ hypobaric bupivacaine $(2 \mathrm{ml})$. Because of this unexpected effect of local anesthetic solution, in the second operation, the technique was changed to intrathecal injection of $12.5 \mathrm{mg}$ hypobaric bupivacaine $(4 \mathrm{ml})$, and the motor and sensory blockade at Th10 was achieved again. The patient reported satisfactory anesthesia each time, and developed no complications. In conclusion, spinal anesthesia can be successful even in cases of severe thoracolumbar kyphoscoliosis. — spinal anesthesia; thoracolumbar kyphoscoliosis

(C) 2005 Tohoku University Medical Press

Patients with spinal abnormalities present unusual challenges for the administration of sedation and anesthesia during surgical and technical procedures. Airway management and respiratory problems are the commonest. Spinal deformities can cause difficulties with regional anesthesia (Kanri et al. 1990; Kleinman 2002). We describe the anesthetic management of cystolithotripsy operation using spinal anesthesia in a patient with extremely severe thoracolumbar kyphoscoliosis.

\section{Case Report}

A 31-year-old man with extreme S-shaped curvature of the thoracolumbar spine and bladder stones was scheduled for cystolithotripsy. His neck movements, including extension, were

Received May 31, 2005; revision accepted for publication August 17, 2005.

Correspondence: Gurayten Ozyurt, Uludag University, School of Medicine, Department of Anesthesiology, 16059 Bursa, Turkey.

e-mail: gurayten@uludag.edu.tr 
restricted, and he was unable to walk because of contractures in his lower extremities. He was restricted to sitting position, and was unable to move unaided (Fig. 1). Fig. 2 shows the roentgenogram of his thoracolumbar spine. The patient was alert and cooperative. His airway was assessed as Mallampati class III (difficult oroteacheal intubation is to be expected in Mallampati class III or IV, which implies the inability to visualize certain pharyngeal structures) (Mallampati et al. 1985). The thyromental distance was $5 \mathrm{~cm}$, also predictive of difficult orotracheal intubation (Hagberg and Boin 1999). Physical examination revealed limited lung capacity and reduced breath sounds bilaterally. Spirometry revealed a forced vital capacity of 1.21 (65\% of predicted) and a forced expired volume in 1 second of 1.151 (88\% of predicted). Routine laboratory tests and chromosome analysis were normal. The patient had been diagnosed as having early-onset idiopathic scoliosis, which had never been treated.

Spinal anesthesia was considered the best

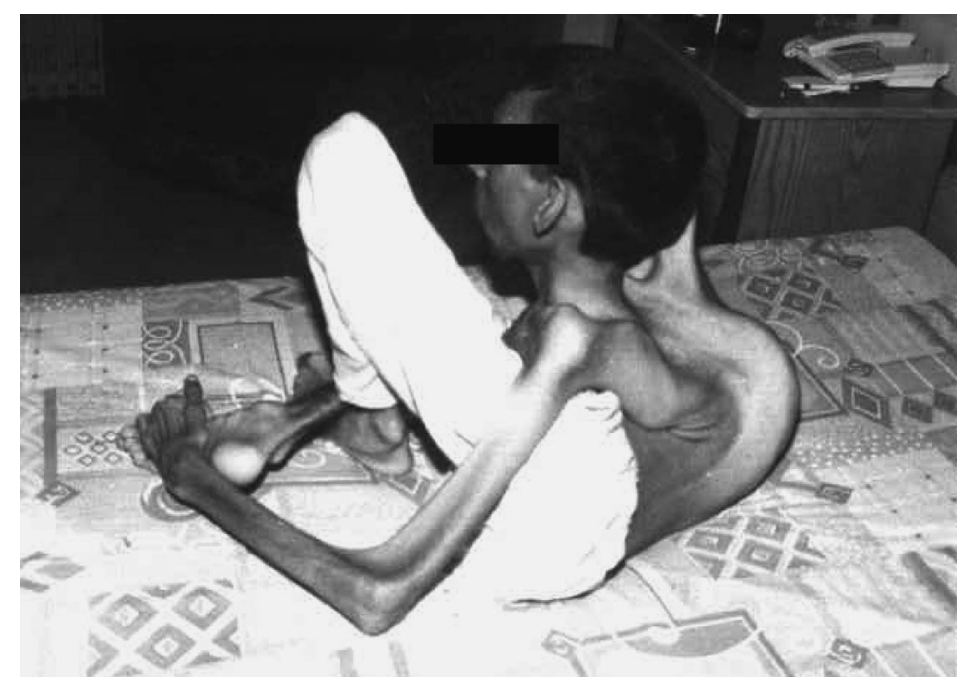

Fig. 1. The patient with severe thoracolumbar kyphoscoliosis.

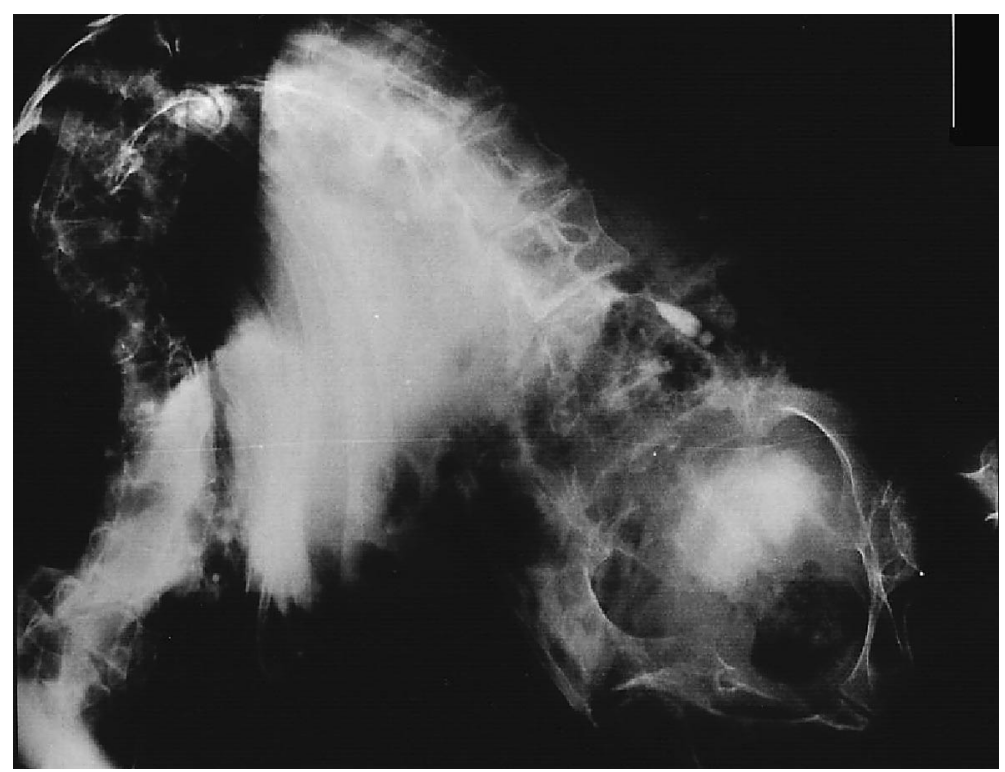

Fig. 2. The roentgenogram of the patient with severe thoracolumbar kyphoscoliosis. 
option for the lithotripsy procedure. The patient was informed about what would be involved in this type of anesthesia, and gave his consent. Preparations were made for airway and circulatory and ventilatory support, in the event of high spinal anesthesia. Oxygen was administered by face mask and the patient was continuously monitored with electrocardiography, pulse oximetry and noninvasive blood pressure assessments. For spinal anesthesia, the patient was placed in right lateral decubitus position. After local anesthetic infiltration, a 25-gauge Quincke spinal needle (Spinocan $^{\circledR}, \mathrm{B} /$ Braun, Melsungen AG, Germany) was inserted at the L3-4 interspace. The needle was slowly advanced toward the dura, and the subarachnoid space was reached after the needle had been advanced $1.5 \mathrm{~cm}$. An intrathecal (i.t.) injection of $6 \mathrm{mg} 0.5 \%$ hyperbaric bupivacaine (Marcaine $0.5 \%$ heavy, Eczacibasi, Istanbul), a local anesthetic agent. Hyperbaric solution (1.2 $\mathrm{ml}$ ) of local anesthetic is of high density compared with cerebrospinal fluid (CSF), was then administered, and the patient was immediately turned to supine position. However, this block was inadequate, with pinprick testing indicating sensory blockade at L1 level on the left side only, and no detectable motor blockade on either side. In normal conditions, with a head-down position, a hyperbaric solution spreads cephalad and a hypobaric anesthetic solution, which is less dense than CSF moves caudad. Head-up position causes a hyperbaric solution to settle caudad and the hypobaric solution ascends cephalad (Kleinman 2002). Similarly, administration of hyperbaric solution in the right lateral decubitus position would produce right sided anesthesia; in this case only the left side was inadequately blocked. We thought that the explanation might be the abnormal curvature of the spine, the rotation and angulation of the vertebral bodies and neural elements, and slightly upward position of its upper part. We therefore decided to reverse the strategy and use hypobaric solution in the same position. A hypobaric solution was prepared by mixing $2.5 \mathrm{ml}$ isobaric bupivacaine (Marcaine 0.5\%, Eczacibasi, Istanbul), $0.2 \mathrm{ml}$ fentanyl (Fentanyl citrate, Abbott Laboratories, Chicago, IL, USA) and $1.3 \mathrm{ml}$ dis- tilled water (4 $\mathrm{ml}$ total volume). The patient was returned to right lateral decubitus position, and an i.t. injection of $6.25 \mathrm{mg}$ hypobaric bupivacaine and $5 \mu \mathrm{g}$ fentanyl ( $2 \mathrm{ml}$ total volume) was administered at the same site using the same size needle. Testing 5 min after this injection confirmed sensory blockade at Th10 and bilateral motor blockade. The patient was then turned to the supine position. Surgical anesthesia was adequate during the entire procedure, which was uneventful and lasted 75 min. The patient's vital signs remained stable throughout. At 180 min after i.t. injection, sensory and motor functions were completely restored. Two days later, the patient was discharged with no complications.

Fifteen months later, the patient was scheduled for the same procedure. There had been no changes in his general clinical condition, and he expressed satisfaction with the quality of anesthesia during the previous procedure, requesting that spinal anesthesia be used again. Again, a 25-gauge Quincke spinal needle (Spinocan ${ }^{\circledR}$, B/Braun, Melsungen AG, Germany) was inserted at the L3-4 spinal interspace and advanced $2 \mathrm{~cm}$ until CSF flow began. Then $12.5 \mathrm{mg}$ hypobaric bupivacaine and $10 \mu \mathrm{g}$ fentanyl (4 $\mathrm{ml}$ total volume) were injected. Testing at $10 \mathrm{~min}$ showed adequate motor blockade and sensory blockade at Th10 on both sides. The patient remained comfortable and hemodynamically stable throughout the 60 min procedure, which was uneventful. Sensory and motor functions were completely restored two hours after the i.t. injection. The patient was discharged the next day with no problems.

\section{Discussion}

Spinal anesthesia is less reliable in the presence of kyphoscoliosis, but successful outcomes have been described (Moran and Johnson 1990; Douglas 1995; Dresner and Maclean 1995; Hatzakorizan et al. 2001). In one case of a patient with severe kyphoscoliosis, an attempt at continuous spinal anesthesia with repeated doses of hyperbaric bupivacaine was unsuccessful, and adequate surgical anesthesia was only achieved by adding isobaric bupivacaine solution (Moran and 
Johnson 1990). Douglas (1995) described an asymmetric block in a patient with marked scoliosis; the patient had incomplete block on the left, but satisfactory spinal block was obtained with hyperbaric bupivacain after the table was tilted to the left. In our patient with severe thoracolumbar kyphoscoliosis, hyperbaric bupivacaine produced an inadequate, unilateral spinal block. We achieved symmetrical sensory and motor blockade with the injection of hypobaric bupivacaine.

The most common technique patient position for spinal anesthesia utilizes the lateral decubitus position, with injection of hyperbaric anesthetic solution via a midline approach. Hyperbaric solutions produce safe and relatively consistent spinal blocks for many types of operations (Sakura 2000). This was our rationale for injecting hyperbaric solution in our first attempt. We suspect that our patient's deformity caused the anesthetic solution to gravitate to the lower end of the spinal column and spread unilaterally. In the supine position, flexion of the hips reduces lumbar curvature and thus the spread of hyperbaric solutions (Fried and Wildsmith 1996). Our patient's hips were permanently flexed due to lower limb contractures, his thoracolumbar spine was severely curved and the upper vertebrae were in slightly upward position. These abnormalities might have altered the normal distribution of hyperbaric solution by changing the contour of the subarachnoid space.

Some studies have suggested that baricity is an important determinant of local anesthetic distribution in the subarachnoid space (Lui et al. 1991; De Wachter et al. 1997). At the first attempt it was difficult to predict the distribution of the local anesthetic solution and we applied the most commonly used technique, but the sensory block achieved was incomplete. Therefore we repeated the spinal block with the hypobaric solution, but still in the right lateral position. We maintained the patient in this position for $5 \mathrm{~min}$, and obtained bilateral surgical anesthesia of adequate duration. We concluded that the hypobaric bupivacaine spread cranially because of the slightly upward position of the vertebra. In the second operation, we changed the technique, increasing the dosage of hypobaric local anesthetic to $12.5 \mathrm{mg}$, and the total volume injected to 4 $\mathrm{ml}$. Probably the greater dose of bupivacaine, greater volume, and lesser density of the anesthetic solution were all important for achieving adequate anesthesia.

This case demonstrates that spinal anesthesia can be successful even in cases of severe thoracolumbar kyphoscoliosis. Intrathecal injection of hypobaric local anesthetic solution may help achieve symmetrical and adequate motor and sensory blockade in patients with extreme spinal deformities or musculoskeletal conditions affecting the spinal column.

\section{References}

De Wachter, D.S., Verdonck, P.R. \& Verhoeven, R.F. (1997) Distribution of marcaine in an in vitro model of the subarachnoid space conforming to actual spinal column geometries. Technol. Health Care, 5, 437-447.

Dresner, M.R. \& Maclean, A.R. (1995) Anaesthesia for Caesarean section in a patient with Klippel-Feil syndrome. The use of a microspinal catheter. Anaesthesia, 50, 807-809.

Douglas, M.J. (1995) Unusual regional block. Can.J. Anaesth., 42, 362-363.

Fried, M.J. \& Wildsmith, J.A.W. (1996) Spinal and epidural anaesthesia-practical aspects. In: International Practice of Anaesthesia, edited by C. Prys-Roberts \& B.R. Brown, Jr., Butterworth-Heinemann, Oxford, pp. 2/139/1-2/139/22.

Hagberg, C. \& Boin, M.H. (1999) Management of the airway: complications. In: Anesthesia and Perioperative Complications, edited by J.L. Benumof \& L.J. Saidman, Mosby, St. Louis, pp. 3-24.

Hatzakorizan, R., Robbins, R. \& Plourde, G. (2001) Spinal anesthesia at the cervicothoracic level. Anesthesiology, 95, 266-268.

Kanri, T., Watanabe, K., Yoshikawa, T., Suzuki, T., Sano, K., Kitano, T., Ninomiya, T., Matsui, T., Fujii, K. \& Takano, K. (1990) Experiences in general anesthesia of patients with scoliosis presupposed to have difficulties in airway maintenance. Shigaku, 78, 377-382.

Kleinman, W. (2002) Spinal, epidural and caudal blocks. In: Clinical Anesthesiology, edited by G.E. Morgan, Jr., M.S. Mikhail, M.J. Murray \& C.P. Larson, Jr., Lange Medical Books, McGraw-Hill, New York, pp. 253-282.

Lui, A.C., Munhall, R.J., Winnie, A.P. \& Selander, D. (1991) Baricity and distribution of lidocaine in a spinal canal model. Can. J. Anaesth, 38, 522-526.

Mallampati, S.R., Gatt, S.P., Gugino, L.D., Desai, S.P., Waraksa, B., Freiberger, D. \& Liu, P.L. (1985) A clinical sign to predict difficult tracheal intubation: A prospective study. Can. Anaesth. Soc. J., 32, 429-434.

Moran, D.H. \& Johnson, M.D. (1990) Continuous spinal anesthesia with combined hyperbaric and isobaric bupivacaine in a patient with scoliosis. Anesth. Analg., 70, 445-447.

Sakura, S. (2000) Factors influencing the level of spinal anesthesia: (I). Characteristics of anesthetic solutions. Masui, 49, 18-25. 